\title{
A vulnerabilidade da infância frente ao excesso de peso: considerações éticas sobre responsabilidades.
} The vulnerability of children against overweight:
ethical considerations responsibilities. La vulnerabilidad de los niños frente al sobrepeso: consideraciones éticas acerca de las responsabilidades.

Caroline Filla Rosaneli ${ }^{1}$ Thiago Rocha da Cunha

RESUMO: A obesidade e sobrepeso na infância e suas consequências clínicas em outras fases da vida estão bem documentadas, porém, este problema emergente de saúde pública envolve questões éticas cujas dimensões necessitam de melhor compreensão. O objetivo deste artigo é discutir o impacto do excesso de peso na infância a partir de uma problematização bioética acerca da responsabilidade das diversas esferas da sociedade - especialmente da família, da mídia e do estado - na proteção deste grupo que é sensivelmente mais vulnerável aos padrões de consumo alimentares não saudáveis. A argumentação desenvolve-se no sentido de que tais medidas de proteção devem ser tomadas a partir de uma responsabilização mútua sustentada no dever do respeito à dignidade das crianças e no compromisso ético com o futuro saudável das próximas gerações.

Palavras-chave: Sobrepeso; Obesidade; Crianças; Responsabilidade Social; Bioética.

\begin{abstract}
Obesity and overweight in childhood and its clinical consequences in other stages of life are well documented, but this emerging public health problem involves ethical issues whose dimensions need better understanding. The objective of this paper is to analyze the impact of excess weight in childhood from a bioethics questioning regarding the responsibility of the various spheres of society - especially the family, the media and the state - in the protection of this group is significantly more vulnerable to standards of unhealthy food consumption. The argument is developed in the sense that such protective measures must be taken from a sustained mutual accountability in the duty of respect for the dignity of children and the ethical commitment to the healthy future of the next generations.
\end{abstract}

Keywords: Overweight; obesity; children; Social Responsibility; Bioethics.

1 Programa de Mestrado em Bioética. Escola de Saúde e Biociências. E-mail: caroline.rosaneli@gmail.com

2 Programa de Mestrado em Bioética. Escola de Saúde e Biociências. E-mail: caixadothiago@gmail.com 
RESUMEN: La obesidad y el sobrepeso en la infancia y sus consecuencias clínicas en otras etapas de la vida son así documentadas, pero este problema emergente de salud pública implica cuestiones éticas cuyas dimensiones necesitan un mejor entendimiento. El objetivo de este trabajo es analizar el impacto del exceso de peso en la infancia a partir de un cuestionamiento bioética sobre la responsabilidad de los distintos ámbitos de la sociedad - especialmente la familia, los medios de comunicación y el Estado - en la protección de este grupo es mucho más vulnerable a las normas del consumo de alimentos poco saludables. El argumento se desarrolla en el sentido de que las medidas de protección deben ser tomadas de una responsabilidad mutua sostenida en el deber de respeto a la dignidad de los niños y el compromiso ético con el futuro saludable de las próximas generaciones.

Palabras clave: El sobrepeso; La obesidad; Infancia; Responsabilidad Social; Bioética.

\section{INTRODUÇÃO}

Segundo a Organização Mundial da Saúde, em 2010, o número de crianças obesas menores de cinco anos era mais de 43 milhões e, além disto, cerca de 92 milhões de crianças, neste mesmo período, estavam em risco de sobrepeso ${ }^{1,2}$. No Brasil, um terço das crianças nesta faixa etária sofre de sobrepeso ou obesidade ${ }^{3}$.

A prevalência mundial de sobrepeso e obesidade infantil aumentou de 4,2\% em 1990 para 6,7\% em 2010. Esta tendência deve chegar a $9 \%$ da população infantil mundial ou cerca de 60 milhões de crianças em $2020^{2}$. Em países desenvolvidos estima-se que a prevalência de excesso de peso aumente cerca de $1 \%$ ao ano, atingindo 15 milhões de crianças e adolescentes ${ }^{4,5}$.

Há uma complexa relação para explicar as rápidas alterações no ganho de peso na infância e adolescência como genética, ambiente social, estilo de vida dos pais e comportamento dietético ${ }^{6,7}$.

Esta condição clínica pode ser analisada como privação dos direitos das crianças e adolescentes, pois os coloca em condição de risco a saúde, impedindo-os de alcançar seu pleno potencial de desenvolvimento humano e tornando-os vulneráveis as condições de saúde e qualidade de vida, o que determina em primeira instância, uma violação dos princípios fundamentais de justiça e direitos humanos ${ }^{8}$.

Ao lançar as bases conceituais da bioética, Potter $^{9}$ indicou que a principal preocupação do campo deveria ser com a sobrevivência saudável das futuras gerações, o que torna a questão do sobrepeso e da obesidade infantil um problema a ser discutido também à luz desta disciplina.

A perspectiva neste artigo é justamente apresentar o impacto da infância vulnerável frente ao excesso de peso a partir de uma problematização ética sobre os riscos relacionados ao consumo e as consequentes responsabilidades pública e individual com a proteção das crianças. 


\section{A infância e sua vulnerabilidade}

De acordo com a Declaração Universal sobre Bioética e Direitos Humanos ${ }^{10}$, a vulnerabilidade, elevada a condição de princípio ético, visa garantir o respeito pela dignidade humana nas situações em relação às quais a autonomia e o consentimento se manifesta insuficiente, como é o caso da infância, portanto, necessita-se assumir a reflexão a partir de uma perspectiva sobre proteção dos vulneráveis.

No Estatuto da Criança e do Adolescente ${ }^{11}$ brasileiro, artigo $3^{\circ}$, é garantido à criança e o adolescente o benefício de gozar de todos os direitos fundamentais inerentes à pessoa humana, sem prejuízo da proteção integral de que trata esta lei, assegurando desta forma, as oportunidades e facilidades para o desenvolvimento físico, mental, moral, espiritual e social, em condições de liberdade e de dignidade.

A consolidação e efetivação dos direitos humanos é uma resposta humana e ética à vulnerabilidade das pessoas nos diferentes ciclos de vida. Frente à vulnerabilidade na infância é necessário assumir, portanto, o cuidado e acolhimento às dificuldades particulares do indivíduo, mas também a responsabilidade da família, da sociedade e do Estado.

Em relação à responsabilidade familiar, Figueiredo ${ }^{12}$ defende que possibilitar que os filhos desenvolvam a obesidade na infância pode ser considerado como negligência, na medida em que os direitos fundamentais da criança e do adolescente são reconhecidos como dever da família e do Estado, com absoluta prioridade, o direito à saúde, à alimentação, além de colocá-los a salvo de toda forma de negligência e discriminação. Ademais, se há negligência para o surgimento da doença, posteriormente poderá ocorrer à discriminação do menor pelo excesso de peso, o que reforça o sobrepeso e obesidade infantil como um problema ético e bioético.

Há uma complexidade ética e cautelosa inclinada em culpar os pais de negligentes quanto aos seus filhos. Em diversos países têm sido observados casos de a família perder a guarda dos filhos por sofrerem de obesidade mórbida e alimentação insegura, gerando discussões ainda em aberto sobre a relação do dever público de proteção das crianças e os limites da intervenção estatal nos riscos e possíveis danos que podem ser ocasionados à criança ${ }^{13}$.

Ainda em relação à responsabilidade pública e familiar, Papalia e Olds ${ }^{14}$ problematizam o fato de que crianças em idade escolar, na maioria das vezes, passam mais tempo longe de casa do que em décadas passadas, mesmo que o lar e a família continuem sendo a parte mais importante do mundo infantil.

De fato, a família é um elo muito importante na formação da criança, para todos os hábitos, sejam eles, saudáveis ou não. A criança observa e aprende tudo a seu redor. Na medida em que os responsáveis pelas crianças é quem fazem as compras e preparo dos alimentos em casa, estes transmitem seus hábitos alimentares às crianças ${ }^{15}$. Por isto, hábitos saudáveis, como o incentivo de 
consumo de frutas, verduras, água, exercício físico, higiene pessoal, e até mesmo cuidados com o planeta será aprendido e absorvido pelo contato com a família. Seja ela qual for a sua configuração. Mas é importante ressaltar que a dimensão familiar não é única onde se define a responsabilidade ou a solução para o problema da obesidade e sobrepeso, uma vez que este é uma questão que envolve toda a sociedade em seus aspectos culturais, econômicos e políticos. Por muitas vezes, é um resultado muito difícil de ser desfeito, e com efeitos em longo prazo, e às vezes, para a vida toda.

A obesidade não traz consequências apenas fisiológicas, mas também está relacionada com a saúde mental e com o bem estar social. Bulling está relacionado ao sobrepeso e à obesidade, e não é só na escola que as crianças são vítimas. Pais e familiares podem tratar a criança de forma preconceituosa, e isto, não facilita a sua recuperação do quadro clínico. A esse respeito, em estudo que investigou sobre o prejuízo causado pela dificuldade de expressão da criatividade no brincar de crianças obesas, as figuras parentais foram vistas como ambivalentes, não suprindo as necessidades afetivas e a dependência dos filhos, mas exigindo sua autonomia. As crianças nesta condição apresentaram características como dependência, passividade e baixa autoestima, além de sinais de timidez, insegurança, vergonha, crença de que eram diferentes e sentimentos de incapacidade e desmotivação ${ }^{16}$.

Todo este problema ético torna-se ainda mais complexo ao considerar o papel da mídia e das tecnologias de comunicação no fomento de alimentação não saudável e da ampliação da epidemia de sobrepeso e obesidade infantil.

\section{O consumo de televisão}

Strasburger ${ }^{17}$ apontou que em apenas $10 \%$ do tempo televisivo assistido por uma criança pode ser considerado como consumo socialmente útil, demonstrando os efeitos nocivos das informações provenientes da televisão para o aumento do comportamento agressivo, da sexualidade precoce, da alimentação e do uso de drogas na população infanto juvenil.

O aparelho de televisão presente em grande parte dos lares tanto dos países desenvolvidos quanto em desenvolvimento constitui uma realidade com a qual é necessário aprender a conviver ${ }^{18}$. A televisão tem sido vista, como maior forma de mídia para infância. Por conta da comunicação de risco que ela propicia ao ser desprotegido de argumentação, a publicidade infantil tem sido alvo de inúmeras tentativas de leis e punição, na busca de uma publicidade infantil mais ética.

No Brasil, o compromisso com a proteção a infância garantindo seu desenvolvimento pleno é um direito assegurado como prioridade na Constituição Federal, no seu artigo $6^{\circ}$ e $227^{\circ}$. É de suma importância criar mecanismos capazes de direcionar cada informação e comunicação de risco numa fonte segura e ética, de forma que sempre sejam usados em seus aspectos positivos, não permitindo que se atrapalhem o crescimento e do desenvolvimento digno e saudável das crianças e adolescente brasileiro ${ }^{19}$. 
Levantamento realizado no Brasil mostrou que as crianças e adolescentes assistem em média 26,46 horas de televisão semanais. Além dos vídeos e videogames, o que certamente se computados, elevariam muito esta média ${ }^{20}$. Outro estudo demonstrou que as crianças passam cerca de 5 horas por dia diante da TV, permitindo que a exposição a mais de 30 segundos de propaganda alimentícia pode influenciar na escolha e hábitos alimentares. Os alimentos representam $27,4 \%$ das propagandas nos dias de semana e $22,3 \%$ nos finais de semana. Alimentos ricos em gordura e açúcares têm participação em $57,8 \%$ dos produtos anunciados e há completa ausência de frutas e hortaliças ${ }^{21}$.

A cada hora diante da TV, a prevalência de sobrepeso pode aumentar até $2 \%$ na infância ${ }^{21,22}$. Nesta fase da vida é recomendado não ultrapassar 2 horas diárias em frente à televisão, mas este número é demonstrado constantemente acima do permitido, e relacionado ao excesso de peso em todas as faixas etárias estudadas ${ }^{23-25}$. O aparelho de TV no quarto pode aumentar em $31 \%$ o risco de um jovem desenvolver sobrepeso ${ }^{18}$.

Na década de 90, Birch e Fischer ${ }^{26}$, discutiram sobre a quantidade de horas das crianças assistindo TV, o conteúdo dos anúncios e a influência no consumo alimentar, e observaram que $80 \%$ das propagandas sobre alimentos eram de baixo valor nutricional, e indivíduos que são expostos a estes anúncios são mais vulneráveis ao consumo de açúcares e gorduras. Em 2012, Boulos e Vikre ${ }^{27}$, demonstraram que a televisão pode aumentar o consumo de alimentos também de forma indireta, além de causar o sedentarismo, pois através da exibição de produtos específicos em programas infantis, podem influenciar as percepções da imagem corporal. Outros estudos indicam uma associação direta entre o tempo gasto assistindo televisão ou outras mídias eletrônicas e peso corporal $^{28}$.

Rosaneli e colaboradores ${ }^{29}$, em um estudo transversal avaliaram o uso da televisão ou computadores durante as refeições e quais os principais alimentos consumidos nesses momentos. A prática das refeições frente à TV ou computador foi relatado em $61,7 \%$ dos casos, e um quarto dos alunos referiu fazer refeições completas (almoço, jantar, café da manhã, ou o somatório de algumas destas refeições) enquanto assistiam à TV ou usavam o computador. O consumo de lanches, guloseimas e doces em geral foram mencionados por $57,1 \%$. Apenas $2,6 \%$ das crianças relataram consumir frutas nesses momentos.

Famílias que possibilitam as refeições frente à televisão consomem $6 \%$ a mais de calorias, 5\% a mais de pizzas e refrigerantes e $5 \%$ a menos de verduras e legumes. Para a cafeína, este aumento é de 12 vezes maior do que para pessoas que se alimentam com o aparelho desligado ${ }^{7}$. Estes dados têm impactado na saúde e longevidade da população. O tempo gasto com TV está relacionado com o metabolismo, indicando um menor gasto energético daqueles que se dedicam a diversas horas diárias em frente $\mathrm{TV}^{30}$.

A era digital está mudando o estilo e comportamento de vida dos jovens, seus relacionamentos com a família e sociedade e sua saúde, sem potencializar o seu desenvolvimento pleno. Há muitas 
dificuldades no diálogo, um paradoxal isolamento no meio de tantos contatos e conexões, em um mundo em constante, e cada vez mais veloz, transformação, que pode ocasionar riscos e problemas à saúde. Riscos individuais como maus hábitos alimentares, sedentarismo, baixo rendimento escolar, baixa autoestima e condutas antissociais são descritas como os principais riscos no desenvolvimento de crianças nesta era digital ${ }^{19}$.

A decisão sobre o tempo de consumo de televisão deveria estar nas mãos dos adultos ${ }^{20}$. Os adultos reconhecem a televisão como fantasia, entretenimento e, frequentemente, irreal. As crianças não são capazes desta discriminação. Aqueles que assistem a muitas horas de televisão acreditam que o mundo é tal como visto na tela ${ }^{17}$.

Portanto, é fundamental que pais, professores, agentes educacionais e todos os responsáveis pela formação moral e ética das crianças, inclusive o Estado, estejam atentos para o quanto o consumo da televisão reflete na saúde infantil.

Embora a Comissão Nacional de Autorregulamentação Publicitária (CONAR) afirme que a autorregulamentação para o público infantil do Brasil esteja entre as mais completas e restritivas do mundo ${ }^{31}$, o fato é que o país se encontra distante daqueles que assumem a regulamentação estatal como uma responsabilidade pública e coletiva ${ }^{32}$.

Portanto, ainda é necessário observar os limites éticos e legais destas situações que envolvem as crianças para não promover perda da qualidade de vida ou mesmo o encurtamento da infância, sobretudo porque o bombardeio publicitário dos meios de comunicação de massa interferem na liberdade de escolha das crianças, sempre vulneráveis e hipossuficientes nas relações de consumo.

\section{Impactos na saúde infantil}

As consequências da obesidade para crianças e adolescentes são graves e podem acarretam precocemente o risco aumentado para doença cardiovascular, problemas psicossociais e distúrbios metabólicos ${ }^{6,33-36}$. Porém, a consequência mais importante é sua persistência na idade adulta juntamente com todos os fatores de risco a ela associados ${ }^{37}$.

Aspectos intelectuais e cognitivos foram investigados em um estudo transversal com escolares obesos e eutróficos, entre oito e 13 anos em São Paulo (SP), analisando o Quociente de Inteligência. O grupo de alunos com peso dentro da normalidade apresentou desempenho significantemente melhor, no teste de inteligência, do que o grupo de obesos. As crianças eutróficas revelaram maior capacidade de adaptação social e melhor velocidade e destreza demonstrando a interferência do excesso de peso no potencial intelectual de crianças $^{38}$.

O exercício físico regular desde a primeira infância torna a criança menos vulnerável aos fatores de risco relacionados ao excesso de peso $^{39,40}$. Estudos demonstram que no Brasil a prevalência de inatividade física entre escolares é de aproximadamente $90 \% \%^{3,41,42}$. O tempo despendido com 


\section{$35 / /$}

atividades físicas de lazer é ainda menor, principalmente nas classes econômicas baixas ${ }^{43}$. Com a atividade física, o indivíduo tende a escolher alimentos menos calóricos ${ }^{44}$.

O excesso de horas no uso do computador e hábitos sedentários potencializa a queda do rendimento escolar, dificultando prevenir os problemas e tantas outras ameaças à saúde desta geração digital ${ }^{19}$.

O ganho de peso desde ao nascimento é um indicador importante para perceber a obesidade na infância. Estudo desenvolvido na Universidade de Harvard ${ }^{45}$, demonstrou que o aumento de altura e peso de uma criança acelerado até os seis meses de idade, indica que ela terá indícios de ser obesa aos cinco anos. Este é um estudo perturbador, porque 'condena' aos seis meses de idade a vulnerabilidade da infância frente ao excesso de peso. Diante disso, o questionamento sobre a responsabilidade familiar e pública torna-se ainda mais premente.

Outro aspecto já indicado neste trabalho é que os sentimentos da criança com excesso de peso acerca da sua aparência física podem causar sofrimento emocional, isolamento por se acharem diferentes do seu grupo, baixa autoestima, o que os leva a uma maior vulnerabilidade à depressão e compensação no alimento em excesso. Desde uma perspectiva ética, para Marques ${ }^{46}$, maltratar o corpo, com práticas nocivas, como a adoção de hábitos não saudáveis, sugere uma profanação de um território sagrado, que deve ser cuidado, cultivado e protegido: o corpo do homem.

Assim, o risco para o excesso de peso na infância potencializa a fragilidade à saúde por toda a vida, e ao considerar que quase um terço das crianças brasileiras menores de cinco anos está desprotegido de segurança, deve-se buscar com mais urgência as medidas de proteção das crianças, sobretudo frente aos impactos em sua saúde associados ao excesso de peso e seus determinantes sociais.

\section{Os riscos no consumo alimentar}

O direito e acesso a alimentação adequada, em qualidade e quantidade, compõe o quadro de proteção da infância saudável, possibilitando a esta fase o crescimento e desenvolvimento adequados. Por outro lado, o consumo alimentar desenfreado, hábitos alimentares incorretos ou comunicação de risco para a infância, potencializam o ganho de peso e suas consequências.

Fatores externos e internos podem contribuir no comportamento alimentar. A unidade familiar e suas características, atitudes dos pais e amigos, valores sociais e culturais, mídia, fast foods, manias alimentares e conhecimento das práticas alimentares, podem ser considerados como a influência externa ao indivíduo. Já nos fatores internos a interferência acontece com as necessidades e características psicológicas, imagem corporal, valores e experiências pessoais, autoestima e preferências alimentares ${ }^{44}$.

A participação da família durante a refeição foi avaliada entre jovens de 9 a 14 anos, e em 16\% 
desta amostra, a família está ausente neste momento ${ }^{6}$.

Hábitos alimentares adquiridos durante a infância influenciarão nas preferências e práticas na ingestão alimentar por toda a vida, e as intercorrências disto, podem ser vistas cada vez mais precocemente ${ }^{47}$.

É importante ressaltar que quanto mais cedo se estabelece bons hábitos alimentares, menor é o risco de apresentar problemas relacionados à obesidade e suas complicações em fases posteriores da vida ${ }^{48}$.

No entanto, o hábito de não se alimentar no café da manhã, de no jantar consumir grande quantidade calórica, ingerir preparações limitadas em grandes porções e excessos de líquidos açucarados são fatores comprovadamente prejudiciais e indutores da obesidade e da condição de risco que o indivíduo se coloca.

A omissão do café da manhã foi associada à obesidade na região Sul do Brasil, entre escolares de oito a 10 anos, podendo significar uma tentativa equivocada de reduzir calorias e alterando o comportamento de alimentos ao longo do dia. A baixa frequência do consumo de leite foi especificamente associada à obesidade ${ }^{49}$. A omissão do café da manhã entre escolares também aparece associada à vulnerabilidade à saúde e o ganho de peso ${ }^{50}$.

Em crianças ingressantes no ensino fundamental provenientes de escolas públicas no Município de Cajamar, SP, encontrou-se um elevado consumo de alimentos considerados não saudáveis (refrigerante, batata frita, bolachas, balas, biscoitos, doces entre outros). Os autores discutem que a infância é um importante grupo para estratégias de prevenção e controle de peso, porque tem maiores possibilidades de sucesso nas ações a serem implementadas e que a escola pode ser o local ideal ${ }^{51}$.

Para Sichieri e colaboradores ${ }^{52}$, a caracterização de padrões de consumo no Brasil é determinada por condições socioeconômica e que, independente delas, mostra-se como fator de proteção para sobrepeso. Por isto, incentivar bons hábitos alimentares, torna o indivíduo menos vulnerável a problemas de saúde.

Na região Sudeste e Nordeste do Brasil moram dois terços da população brasileira, e apresentam os extremos do desenvolvimento desigual do país. A relação entre fatores socioeconômicos e demográficos, e os padrões de consumo de alimentos e sobrepeso destas regiões foram analisados e concluíram que o tamanho da família, variáveis socioeconômicas, estado civil, cor e a região de residência podem interferir no consumo dos brasileiros ${ }^{52}$.

A condição socioeconômica menos favorecida tem sido relacionada com a qualidade e quantidade dos alimentos de alta densidade energética e de baixo custo ${ }^{21,53}$. A qualidade da dieta dos indivíduos parece ser melhor entre os com maior renda e escolaridade ${ }^{54}$. Resultados obtidos 


\section{$37 / /$}

por Guedes e colaboradores ${ }^{55}$, revelaram prevalências associadas ao sobrepeso e à obesidade com valores progressivamente maiores de acordo com posições econômicas mais elevadas.

O nível socioeconômico desempenha segundo Nunes, Figueiroa e Alves ${ }^{43}$, uma maior influência na gênese da obesidade do que os fatores étnicos e geográficos. Apesar de a obesidade ser mais intensa nos grupos populacionais economicamente mais favorecidos, atinge infalivelmente todas as idades, sexo e classes sociais. Estes achados reforçam a percepção de que a fragilidade nas escolhas e acesso aos alimentos pode tornar este público ainda mais vulnerável.

\section{Educação, proteção e cuidado}

A Convenção sobre os Direitos da Criança ${ }^{56}$ trouxe a reflexão e consciência à sociedade da responsabilidade que temos com a infância como geração futura e como grupo vulnerável. No entanto, esforços ainda são necessários na proteção sobre os direitos das crianças.

O direito humano a alimentação adequada (DHAA) é o direito de cada indivíduo desde o seu nascimento, de ter acesso físico e econômico, ininterruptamente, à alimentação adequada ou aos meios para obter estes alimentos, sem comprometer os recursos para obter outros direitos fundamentais, como saúde e educação. Este direito humano, fundamental e social está previsto nos artigos $6^{\circ}$ e $227^{\circ}$ da Constituição Federal, definido pela Lei Orgânica de Segurança Alimentar e Nutricional $1^{57}$, que prevê ainda que segurança alimentar e nutricional compreende a realização do direito de todos ao acesso regular e permanente a alimentos de qualidade, em quantidade suficiente, sem comprometer o acesso a outras necessidades essenciais.

Considerando o direito à alimentação adequada como fundamental, a Declaração Universal dos Direitos Humanos aponta em seu artigo XXV que toda pessoa tem direito a um padrão de vida capaz de assegurar a si e a sua família saúde e bem estar, inclusive alimentação ${ }^{58}$.

É dever da família, da sociedade e do Estado assegurar à criança, ao adolescente e ao jovem, com absoluta prioridade, o direito à vida, à saúde, à alimentação, à educação, ao lazer, à profissionalização, à cultura, à dignidade, ao respeito, à liberdade e à convivência familiar e comunitária. Este direito humano, fundamental e social está definido pela Lei Orgânica de Segurança Alimentar e Nutricional, bem como no artigo 11 do Pacto Internacional de Direitos Econômicos, Sociais e Culturais ${ }^{59}$ e outros instrumentos jurídicos internacionais.

O Plano Nacional de Enfrentamento das Doenças Crônicas 2011-2022 $2^{60}$ pretende atuar no combate e na redução dos principais fatores de risco a doenças como sedentarismo, tabagismo, alimentação inadequada, obesidade e excesso de peso. Ele sinaliza o desafio no desenvolvimento de ações e pesquisas no controle e prevenção, inclusive com crianças, porque estão associadas ao estilo de vida sedentário e alimentação inadequada, desde o início da vida, desfavorecendo seu crescimento e desenvolvimento em potencial. 
A escola tem sido alvo do direcionamento das práticas alimentares educativas e uma oportunidade de atuação e envolvimento para ações eficazes na prevenção da obesidade infanto-juvenil. Porém, têm sido observados resultados modestos, e estudos mostram que é necessário inovar para obtenção de melhores estímulos na autonomia do sujeito para adoção de práticas alimentares adequadas e saudáveis ${ }^{47,61-64}$.

Para Nóbrega ${ }^{65}$, a escola tem que dialogar sobre o papel do corpo e sua relação com o processo cognitivo, com conexões e múltiplas entradas, com novos territórios a serem explorados, espaços e lugares que acolham a corporeidade, diminuindo desta forma, a barreira de compreensão do corpo na infância e a autonomia do sujeito a cumprir normas ou imposições sociais, ou aceitação de sua existência corporal.

A política da escola pode promover ou desencorajar dietas saudáveis e atividade física. A inclusão curricular ou atitudes presenciais fazem-se necessárias para que mudanças no hábito alimentar e seu reflexo no perfil nutricional da população possam ser melhorados.

O pressuposto é que professores capacitados com informações de saúde podem se tornar agentes transformadores do comportamento alimentar dos alunos ${ }^{66}$. O professor pode ser um modelo de estímulo da aceitação de alimentos ${ }^{67}$.

Em Portugal, um programa piloto de intervenção para pais de crianças com problemas de obesidade, teve o intuito de sensibilizar e envolver os pais no processo de educar os filhos para a saúde, contribuindo para a promoção da qualidade de vida e do bem-estar da criança, bem como proporcionar o seu desenvolvimento pleno e harmonioso ${ }^{68}$. No Brasil, um programa educacional para prevenção cardiovascular dirigida às crianças em idade escolar mostrou eficácia em reduzir risco a saúde de seus pais, especialmente nas categorias intermediário e alto risco, capacitando e utilizando as crianças como agentes de proteção familiar ${ }^{69}$.

Quando se trata uma criança obesa, se trata a família, pois aqueles que convivem com ela, devem estar cientes deste processo de educação, proteção e cuidado da saúde. Ao se questionar para qualquer indivíduo o que é alimentação saudável, muitos terão uma resposta satisfatória, mas na prática, poucos alcançarão o mesmo resultado.

A infância é uma fase importante para a formação de hábitos e de caráter. Privar a criança de seus desejos é perder a possibilidade de uma relação harmoniosa por toda a vida, porém devemos deixar a criança ciente dos riscos de consumo inadequados durante este ciclo de vida.

Para conscientizar a família e fazer com que todos estejam de acordo com as pequenas e constantes mudanças é um longo processo. Quando um responsável pela criança procura uma consulta para seu filho, nem sempre o sujeito está pronto a assumir a mudança, e às vezes passa a responsabilidade ao seu filho. Criança ou adolescente precisa de regras, e de apoio. Perder peso não é simples, e a manutenção desta perda também não é, mas é possível. Grande parte do sucesso 
é saber lidar com seus medos e ansiedades, e ter apoio de um bom profissional.

A criança não tem poder de compra (na sua maioria), e por isto, ela pode aprender a lidar com limites para o consumo. Dizer que não pode consumir determinado produto é frustrante e sem compreensão para uma criança. Hoje, levar lanche ou fruta para escola para ser castigo e vergonhoso perante os amigos. Por isto, e em outras ações diárias, a família tem papel importante em orientar - e ser orientada - sobre a forma correta de escolhas e de exposição a estas condutas perante a sociedade.

As novas gerações podem viver menos tempo do que as anteriores, contrariando a ordem natural da evolução da espécie humana. A epidemia da obesidade na infância, e também em todos os ciclos da vida, tornam os homens vulneráveis a sua existência. Os pais, professores e todos aqueles sistematicamente próximos das crianças precisam ser alertados para a necessidade de deixarem de perpetuar seus maus hábitos alimentares e isto tem que acontecer de uma forma pedagógica, não punitiva, na medida em que requer multifatores para que haja sucesso e suporte efetivo ${ }^{44}$.

Enfim, há necessidade de trabalho interdisciplinar que combina elementos cultural, social e ético para o compromisso com o futuro da infância. Deste modo, os problemas da criança devem integrar a família, a sociedade e o Estado no compromisso e na responsabilidade com a saúde, a educação, a justiça para as crianças ${ }^{8,70}$.

\section{CONSIDERAÇÕES FINAIS}

O aumento da prevalência do excesso de peso e de suas complicações representa um importante desafio aos serviços e às políticas públicas de saúde, mas também uma questão ética para todas as esferas da sociedade, na medida em que acarreta forte impacto social, econômico e na qualidade de vida desta e das futuras gerações.

A quantidade de refeições, o comportamento de consumo de alimentos e a atividade física dos indivíduos são desde cedo aprendidos. Se o ambiente que a criança está inserida lhe proporciona educação, consumo adequado em quantidade e qualidade de alimentos, atividades e movimentos físicos, isto poderá prevenir complicações futuras à saúde e respeito à vida. Por isso, são necessárias ações focadas na solução do excesso de peso e sua associação com outras doenças, na tentativa de minimizar a exposição dos riscos à saúde das crianças.

Modelos estereótipos do corpo como objeto numa luta de aceitação da condição do olhar de outros sobre seu corpo, faz parte da integração da sociedade, legitimadas e impostas como padrões como uma lógica de classificação. Na infância este modelo torna-se perverso e excludente de uma infância digna e justa. É preciso, deste modo, atentar aos padrões de saúde e estéticos que são dialogados e permear o bom senso e justiça no tratamento destas causas.

De fato, os corpos passam por processos de modelação cultural, assumindo distintos significados 
em distintos espaços sociais e diferentes épocas. O bebê encorpado, 'gordinho' é aceito por parecer apresentar característica de volume de comida e saúde. Um ano depois de sua permeabilidade no mundo social e alimentar; ele já pode ser condenado a dietas restritivas em calorias, por ganhar com facilidade peso, mas ainda permanece socialmente adequado. Porém, ao longo de sua primeira infância, se este modelo corpóreo lhe é permanente, passará a sofrer, e às vezes, por todo a sua vida, de estigmas sociais sobre a representação do seu corpo.

Também por isso não se pode esperar para desenvolver ações para a alimentação adequada na infância. Tais medidas devem ser tomadas a partir de uma responsabilização mútua entre os diversos setores da sociedade para garantir cidadania com respeito à vida, à dignidade e à proteção dos vulneráveis.

\section{REFERÊNCIAS BIBLIOGRÁFICAS}

1. Word Health Organization. Obesity and Overweight. WHO [Internet]. 2011 Março [acesso em 22 Maio 2015]; Disponível em: http://www.who.int/mediacentre/factsheets/fs311/en/.

2. de Onis M, Blössner M, Borghi E. Global prevalence and trends of overweight and obesity among preschool children. Am J Clin Nutr. 2010;92(5):1257-64.

3. IBGE. Pesquisa Nacional de Saúde do Escolar 2009, Rio de Janeiro, Brasil. 138p.

4. Lobstein T, Baur L, Uauy R. Obesity in children and young people: a crisis in public health. Obes Rev. 2004;5(Suppl 1):4-104.

5. Nishida C, Uauy R, Kumanyika S, Shetty P. The joint WHO/FAO expert consultation on diet, nutrition and the prevention of chronic diseases: process, product and policy implications. Public Health Nutr. 2004;7(1A):245-50.

6. Taveras EM, Berkey CS, Rifas-Shiman SL, Ludwig DS, Rockett HR, Field AE, et al. Association of consumption of fried food away from home with body mass index and diet quality in older children and adolescents. Pediatrics. 2005;116(4):e518-24.

7. Coon KA, Goldberg J, Rogers BL, Tucker KL. Relationships between use of television during meals and children's food consumption patterns. Pediatrics. 2001;107(1): E7.

8. Sarmiento P. Bioética e infancia: compromiso ético con el futuro. Pers. Bioét. 2010;4(1):10-29.

9. Potter VR. Bioethics: bridge to the future. Englewood Cliffs: Prentice Hall; 1971.

10. Organização das Nações Unidas para a Educação, a Ciência e a Cultura. Universal Declaration on Bioethics and Human Rights [Internet]. 2005 Oct. [acesso em 22 Maio 2015]; 


\section{$41 / /$}

Disponível em: http://www.unesco.org/new/en/social-and-human-sciences/themes/bioethics/ bioethics-and-human-rights/

11. Brasil. Estatuto da Criança e do Adolescente: disposições legais pertinentes. Senado Federal: Brasília; 1990.

12. Figueiredo T. Obesidade infantil decorrente de negligência parental [Internet]. 2012. [acesso em 01 jul 2014]; Disponível em: http://www.jurisway.org.br/v2/dhall.asp?id_dh=8346

13. Merry MS, Voigt K. Risk, harm and intervention: the case of child obesity. Med Health Care Philos. 2014;17(2):191-200.

14. Papalia DE, Olds SW. Desenvolvimento Humano. 7a ed. Porto Alegre: Artmed; 2000.

15. Gambardella AMD, Frutuoso MFP, Franch C. Prática alimentar de adolescentes. Rev Nutr. 1999;12(1):55-63.

16. Mishima FKT, Barbieri V. O brincar criativo e a obesidade infantil. Estudos de Psicologia. 2009;14(3):249-255.

17. Strasburger VC. Os adolescentes e a mídia: Impacto Psicológico. Porto Alegre: Artmed; 1999.

18. Doria-Filho U, Pires JMA. Mídia televisiva: impacto sobre a criança e o adolescente. [Internet]. 2012 [acesso em 02 Maio 2015]; Disponível em: http://www.sbp.com.br/show_item2. cfm?id_categoria $=21 \&$ id detalhe $=2901 \&$ tipo_detalhe $={ }_{S}$

19. Eisenstein E, Estefenon SB. Geração digital: riscos das novas tecnologias para crianças e adolescentes. Revista Hospital Universitário Pedro Ernesto UERJ. 2011;(10):42-53.

20. Gomide PIC. Crianças e adolescentes em frente à TV: o que e quanto assistem de televisão. Psicol. Argum. 2002;19(30):17-28.

21. Almeida SS, Nascimento PCB, Quaioti TCB. Quantidade e qualidade de produtos alimentícios anunciados na televisão brasileira. Rev. Saúde Públ. 2002;36(3):353-355.

22. Dietz WH, Gortmaker SL. Do we fatten our children at the television set? Obesity and television viewing in children and adolescents. Pediatrics 1985; 75(5):807-12.

23. Neville L, Thomas M, Bauman A. Food advertising on Australian television: the extent of children's exposure. Health Promot Int. 2005;20(2):105-12.

24. Dennison BA, Erb TA, Jenkins PL. Television viewing and television in bedroom 
associated with overweight risk among low-income preschool children. Pediatrics. 2002;109(6):1028-35.

25. Lowry R, Wechsler H, Galuska DA, Fulton JE, Kann L. Television viewing and its associations with overweight, sedentary lifestyle, and insufficient consumption of fruits and vegetables among US high school students: differences by race, ethnicity, and gender. J Sch Health. 2002;72(10): 413-21.

26. Birch LL, Fisher JO. Development of eating behaviors among children and adolescents. Pediatrics. 1998;101(3 Pt 2):539-49.

27. Boulos R, Vikre EK, Oppenheimer S, Chang H, Kanarek RB. ObesiTV: how television is influencing the obesity epidemic. Physiol Behav. 2012;107(1):146-53.

28. Strasburge VC. Children, adolescents, obesity, and the media. Pediatrics. 2011;128(1):201-8.

29. Rosaneli CF, Oliveira AA, Oliveira ERN, Nakashima ATA, Auler F. Televisão, computadores e consumo de alimentos: uma relação indigesta. In: Anais do I Congresso Internacional em Estudos da Criança; Braga, Portugal. CD; 2008.

30. Klesges RC, Shelton ML, Klesges LM, Effects of television on metabolic rate: potential implications for childhood obesity. Pediatrics. 1993;91(2): 281-6.

31. Conselho Nacional de Autorregulamentação Publicitária. Publicidade e Criança: Comparativo Global da Legislação e da Autorregulamentação. São Paulo: ABAP; 2013.

32. Veiga EM, Pannunzio MIR, Cunha TR, Garrafa V. A legitimidade da intervenção estatal na publicidade de produtos sujeitos à vigilância sanitária. Rev. de Direito Sanit. 2011;12(2):91111.

33. Associação Brasileira para o Estudo da Obesidade e da Síndrome Metabólica. Diretrizes Brasileiras de Obesidade 2009/2010. 3a ed. Itapevi, SP: AC Farmacêutica; 2009.

34. Wein LM, Yang Y, Goldhaber-Fiebert JD. Assessing screening policies for childhood obesity. Obesity (Silver Spring). 2012;20(7):1437-43.

35. Monteiro P, Victora C, Barros F. Social, familial, and behavioral risk factors for obesity in adolescents. Rev Panam Salud Publica. 2004;16(4):250-8.

36. Oliveira CIL, Fisberg M. Obesidade na infância e adolescência: uma verdadeira epidemia. Arq Bras Endocrinol Metab. 2003; 47(2):107-108. 
37. Organização Mundial da Saúde. Obesidade: Prevenindo e Controlando a Epidemia Global. São Paulo: Roca, 2004.

38. Campos ALR, Sigulem DM, Moraes DEB, Escrivão MAS, Fisberg M. Quociente de inteligência de crianças e adolescentes obesos através da escala Wechsler. Rev. Saúde Públ. 1996; 30(1):85-90.

39. Gidding SS, Lichtenstein AH, Faith MS, Karpyn A, Mennella JA, Popkin B, et al. Implementing American Heart Association pediatric and adult nutrition guidelines: a scientific statement from the American Heart Association Nutrition Committee of the Council on Nutrition, Physical Activity and Metabolism, Council on Cardiovascular Disease in the Young, Council on Arteriosclerosis, Thrombosis and Vascular Biology, Council on Cardiovascular Nursing, Council on Epidemiology and Prevention, and Council for High Blood Pressure Research. Circulation. 2009;119(8):1161-75.

40. Santos MG, Pegoraro M, Sandrini F, Macuco EC. Risk factors for the development of atherosclerosis in childhood and adolescence. Arq Bras Cardiol. 2008;90(4): 276-283.

41. da Silva RC, Malina RM. Level of physical activity in adolescents from Niteroi, Rio de Janeiro, Brasil. Cad. Saude Publ. 2000;16(4):1091-7.

42. Silva MA, Rivera IR, Ferraz MR, Pinheiro AJT, Alves SWS, Moura AA, et al. Prevalence of cardiovascular risk factors in child and adolescent students in the city of Maceio. Arq Bras Cardiol. 2005;84(5):387-92.

43. Nunes MM, Figueiroa JN, Alves JG. Overweight, physical activity and foods habits in adolescents from different economic levels, Campina Grande (PB). Rev Assoc Med Bras. 2007;53(2):130-4.

44. de Mello ED, Luft VC, Meyer F. Individual outpatient care versus group education programs. Which leads to greater change in dietary and physical activity habits for obese children?. J Pediatr. 2004;80(6): 468-74.

45. Taveras E, Rifas-Shiman SL, Sherry B, Oken E, Haines J, Kleinman K, Rich-Edwards JW, Gillman MW. et al. Crossig growth percentiles in infancy and risk of obesity in childhod. Arch Pediatr Adolesc Med. 2011;165(11):993-998.

46. Marques CP. A crise do corpo na sociedade contemporânea: uma reflexão à luz da filosofia e da bioética. Rev. Bioethikos. 2012;6(4):416-421.

47. Perez-Rodrigo C, Aranceta J. School-based nutrition education: lessons learned and new perspectives. Public Health Nutr. 2001;4(1A):131-9. 
48. Organização Panamericana de Saúde. Doenças crônico-degenerativas e obesidade: estratégia mundial sobre alimentação saudável, atividade física e saúde. Brasília; 2003.

49. Triches RM, Giugliani ER. Obesity, eating habits and nutritional knowledge among school children. Rev. Saude Publ. 2005;39(4):541-547.

50. Ortega RM, Requejo AM, López-Sobaler AM, Quintas ME, Andrés P, Redondo MR, et al. Difference in the breakfast habits of overweight/obese and normal weight schoolchildren. Int J Vitam Nutr Res. 1998;68(2):125-32.

51. Mondini L, Levy RB, Saldiva SRDM, Venâncio SI, Aguiar JÁ, Stefanini MLR. Prevalência de sobrepeso e fatores associados em crianças ingressantes no ensino fundamental em um município da região metropolitana de São Paulo, Brasil. Cad. Saúde Publ. 2007;23(8):18251834.

52. Sichieri R, Castro JF, Moura A. Factors associated with dietary patterns in the urban Brazilian population. Cad. Saude Publ. 2003;19(Suppl 1):S47-53.

53. Drewnowski A, Darmon N. The economics of obesity: dietary energy density and energy cost. Am J Clin Nutr. 2005;82(Suppl 1):265S-273S.

54. Barros MBA, Carandina LCC, Goldbaum M. As dimensões da saúde: inquérito populacional em Campinas, São Paulo: Hucitec, 2008.

55. Guedes DP, Paula IG, Guedes JERP, Stanganelli LCR. Prevalência de sobrepeso e obesidade em crianças e adolescentes: estimativas relacionadas ao sexo, idade e classe socioeconômica. Rev Bras Ed Fis Esporte. 2006;20(3):151-163.

56. Organização das Nações Unidas. Declaración de los Derechos del Niño. Genebra, 20 nov 1959.

57. Lei ${ }^{\circ} 11.346$ de 15 de setembro de 2006. Dispõe a Lei Orgânica de Segurança Alimentar e Nutricional. Diário Oficial da União,18 Set 2006.

58. Organização das Nações Unidas. Declaração Universal dos Direitos Humanos, Paris, 10 Dez 1948.

59. Decreto ${ }^{0}$ 591de 6 de julho de 1992. Dispõe sobre o Pacto Internacional de Direitos Econômicos, Sociais e Culturais. Diário Oficial da União, 7 jul 1992.

60. Ministério da Saúde. Plano Nacional de ações estratégicas para o enfrentamento das doenças crônicas não transmissíveis no Brasil 2011-2022 [Internet]. Brasília: Ministério da Saúde, 2011 [acesso em 19 out 2011]. Disponível em: http://bvsms.saude.gov.br/bvs/publicacoes/plano_ 
acoes_enfrent_dent_2011.pdf

61. Ministério do Desenvolvimento Social e combate a fome. Marco de Referência de Educação Alimentar e Nutricional para as Políticas Públicas [Internet]. Brasília: Secretaria Nacional de Segurança Alimentar e Nutricional, 2012 [acesso em 12 jun 2012]. Disponível em: http://www.ideiasnamesa.unb.br/files/marco_EAN_visualizacao.pdf

62. Vasquez F, Andrade M, Rodríguez MP, Salazar G. Effect of educational nutrition program on the energy and macronutrients intake of preschoolers attending Junji day care centres in the eastern sector of Santiago, Chile. Arch Latinoam Nutr. 2008;58(3):241-8.

63. Sharma M. School-based interventions for childhood and adolescent obesity. Obes Rev. 2006;7(3):261-9.

64. Robinson TN. Does television cause childhood obesity? JAMA. 1998; 279(12):959-960.

65. Nóbrega TP. Qual o lugar do corpo na educação? Notas sobre conhecimento, processos cognitivos e currículo. Educ. Soc. 2005;26(91):599-615.

66. Davanço GM, Taddei JAAC, Gaglianone CP. Conhecimentos, atitudes e práticas de professores de ciclo básico, expostos e não expostos a Curso de Educação Nutricional. Rev. Nutri. 2004;17(2):177-184.

67. Bizzo MLG, Leder L. Educação nutricional nos parâmetros curriculares nacionais para o ensino fundamental. Rev. Nutri. 2005;18(5):661-667.

68. Oliveira F, Soares L. Programa piloto de intervenção para pais de crianças com problemas de obesidade. Psicologia, Saúde \& Doenças. 2011;12(2):197-211.

69. Fornari LS, Giuliano I, F Azevedo, Pastana A, Vieira C, Caramelli B. Children First Study: how an educational program in cardiovascular prevention at school can improve parents' cardiovascular risk. Eur J Prev Cardiol. 2013;20(2):301-309.

70. Cortes LC. Moral de mercado versus seguridad alimentaria: una aproximación desde la ética del bien común. Acta bioeth. [online] 2001;7(2):233-248.

Artigo apresentado em 02-09-15

Artigo publicado no sistema em 00-00-16

Artigo aprovado em 30-06-16 